\title{
Evaluation of the Black Sea Land Based Sources of Pollution the Coastal Region of Turkey
}

\author{
Levent Bat ${ }^{*}$, Oylum Gökkurt, Murat Sezgin, Funda Üstün and Fatih Sahin
}

Sinop University Fisheries Faculty 57000 Sinop, Turkey

\begin{abstract}
The Black Sea receives large quantities of unregulated and uncontrolled fresh water with drawl for irrigation purposes, hydro and thermal power generation and the use of coastal areas for permanent human settlements; shipping; and untreated domestic, industrial and agricultural wastes drain into the sea via the rivers or directly. In spite of this, research on the heavy metal pollution in marine biota of the Black Sea is limited.

This review prepared to be informed of the urban sewage pollution loads and heavy metal concentrations of Turkish coasts of Black Sea. The urban sewages and heavy metals currently effective in Turkey coasts of the Black Sea so as to bring up the levels of land based sources of pollution with rivers and streams in the sea. The Black Sea has a special importance because of its being a sea that receives two large rivers, Kizilirmak and Yesilirmak and in this investigation we can show that Yesilirmak has higher amount of discharge then other rivers. This investigation was carried out with indicate to determine the aid of land-based sources and marine activities to the Black Sea, bringing up its present state.

Total chrome and cadmium concentrations are higher then other heavy metals streams and rivers because of high amount of industrial discharges. The data presented in investigation on the heavy metal contamination of marine organisms were different depending on pollution sources, element and species. According to the evaluation of inventories, the results are rising year by year.
\end{abstract}

Keywords: Black Sea, marine pollution, heavy metal, land based sources.

\section{INTRODUCTION}

Marine pollution may be defined as:

' $\ldots$ the introduction by man, directly or indirectly, of substances or energy to the marine environment resulting in such deleterious effect as harm to living resources; hazards to human health; hindrance of marine activities including fishing; impairment of the quality for use of seawater; and reduction of amenities' $[1,2]$.

Balkas et al. [3] pointed out that the oceanography of the Black Sea has been relatively well documented. The same, however, cannot be said for documentation of the levels of marine pollution and the regions that are affected by various human activities, especially in coastal areas [3]. Although, the Turkish coastal regions of the Black Sea are relatively poor in the metal releasing industrial activities, mining and agricultural activities on land may be an important source in the delivery of some metal pollutants. The Black Sea receives large quantities of unregulated and uncontrolled fresh water with drawl for irrigation purposes, hydro and thermal power generation and the use of coastal areas for permanent human settlements; shipping; and untreated domestic, industrial and agricultural wastes drain into the sea via the rivers or directly. In spite of this, research on the heavy metal pollution in marine biota of the Black Sea is very limited. Moreover, corresponding data on the pollution state of the Black Sea off Turkey are rare.

*Address correspondence to this author at the Sinop University Fisheries Faculty 57000 Sinop, Turkey; E-mail: leventbat@gmail.com

\section{THE STUDY AREA}

The Black Sea is the world's largest inland sea and widely perceived to be polluted. Almost one-third of the entire land area of continental Europe drains into it and the Black Sea environment have suffered degradation from the waste from approximately 17 countries (Fig. 1).

The Black Sea is located between the latitudes $40^{\circ} 55^{\prime}$ and $46^{\circ} 42^{\prime} \mathrm{N}$ and the longitudes $27^{\circ} 27^{\prime}$ and $41^{\circ} 42^{\prime} \mathrm{E}$. The Black Sea has historically been one of the most biologically and ecologically productive marine ecosystem in the world.

\section{Evaluation of Urban Sewage Effects to the Turkish Coasts of the Black Sea}

In the Black Sea, some cities use the sewerage system directly but most of the small settlement areas used septic tanks. On the other hand, present sewerage systems show also variety such as combined or separate system (Fig 2). Ordu, Giresun city centres have separate sewerage systems where Sinop, Trabzon and Zonguldak have combined systems but only Samsun city centre have both combined and separate sewerage system [4].

Many industrial untreated industrial and agricultural wastes drain into the sea. Table 1 shows that annual load of pollutants from Turkish Black Sea coast.

Domestic discharge is the greatest source of organic matter discharged into coasts. In Turkey, many towns and cities situated on the coast, however, sewage is discharged untreated. Organic matter is an important nutrient, as it is a source of food for many benthic invertebrates in the marine ecosystem. 


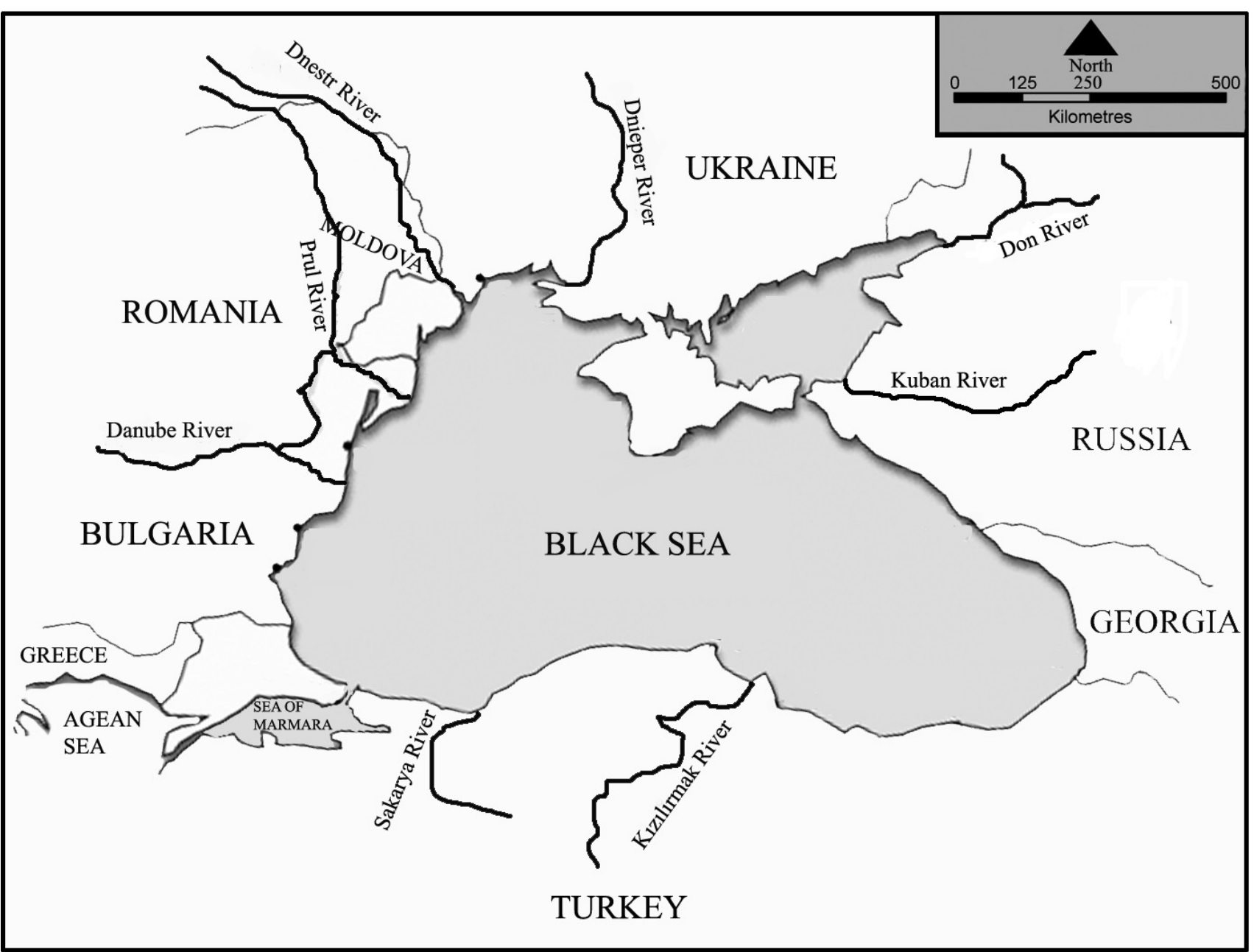

Fig. (1). The Black Sea and its discharge points.

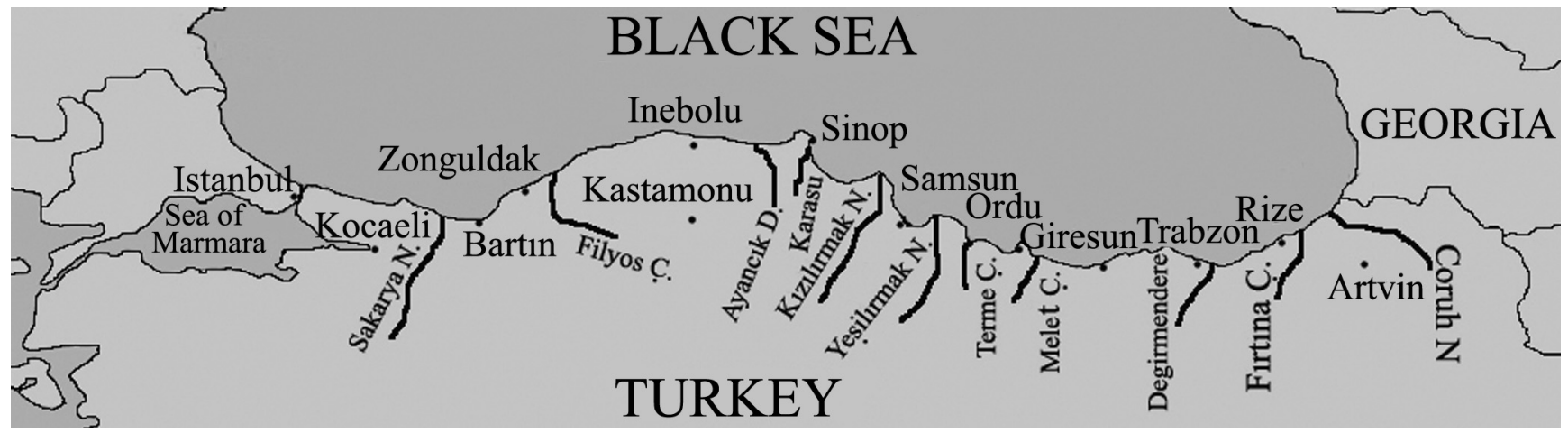

Fig. (2). Black Sea coastal towns and major rivers location points.

For example, Sinop is the smallest city and located on the Southern coasts of the Black Sea. Gökkurt [7] showed that some pollutant loads from Sinop city domestic discharge point to the Black Sea coast (Fig. 3) and Table 2 shows participation of pollutants load from Turkish coast of the Black Sea and especially in Samsun, total load of pollution higher than other cities of Turkish coast of Black Sea (Table 2). The calculations were done according to the flow of the discharge 52 litres per second [8]. Some of the parameters are exceeded the "Turkish Environmental Regulations" criteria. In the last decade, the local population in Sinop is about 30000; however the population increases up to 80000 in summer. Thus, untreated domestic wastes and human activity along the coastal zone increase in summer and probably give rise to high pollution.

In Fig. (3), in Sinop coasts suspended solid matter concentrations is very high respect to Turkish Environmental Regulation, 2004 -General Quality Criteria of Marine. The reason of this situation is estimated that untreated domestic discharges and erosion problem in coastal zone of Sinop peninsula. 
Table 1. Annual Load of Pollutants from Rivers, Streams and Cities (Located in the Coast of Turkey) to Black Sea Coast of Turkey (TSS: Total Suspended Solid, BOD: Biologic Oxygen Demand, COD: Chemical Oxygen Demand, o-P: Ortho Phosphate, Total P: Total Phosphate) [5]

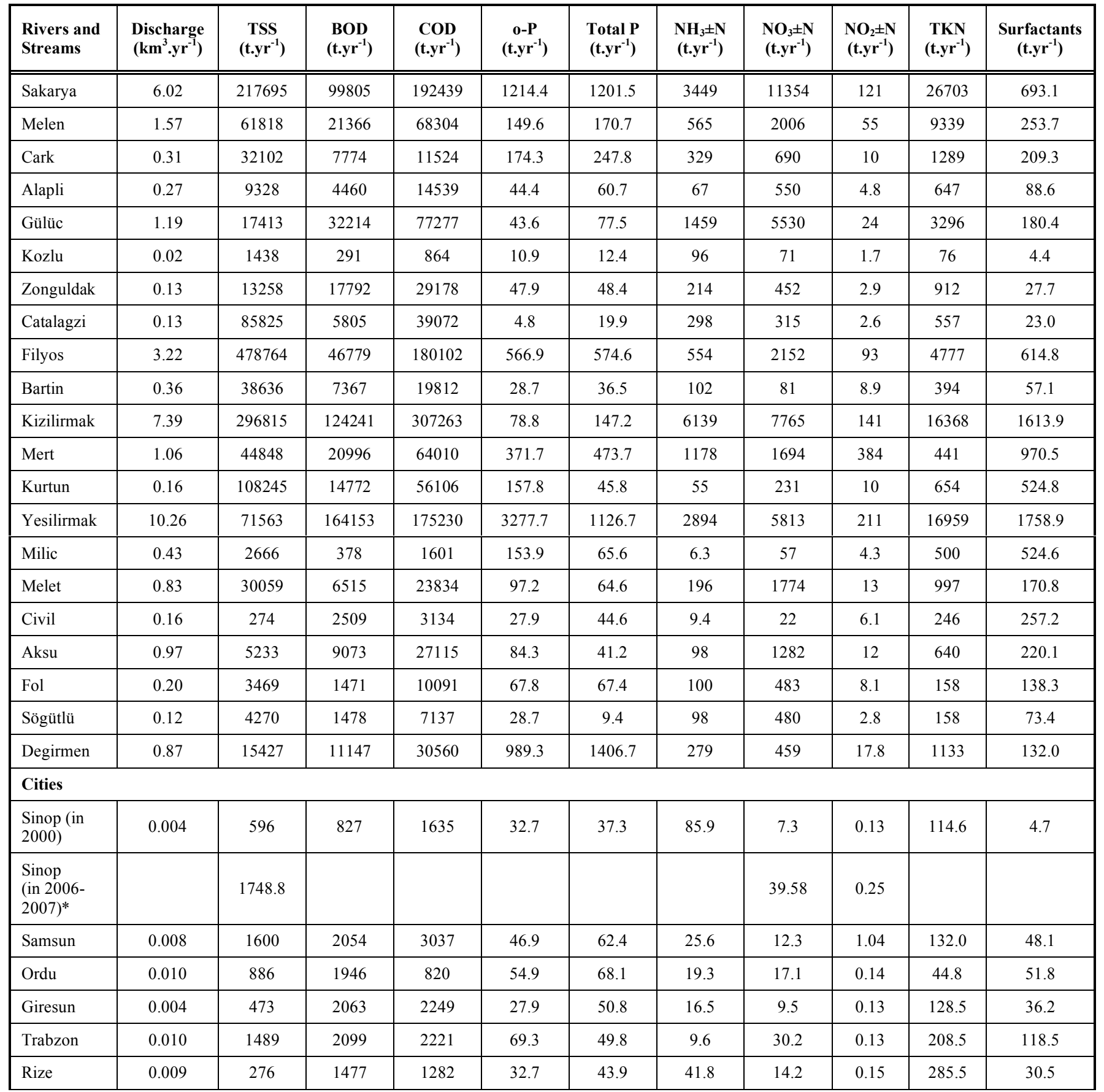

*Data from [6].

Sewage discharges are being dumped in many in coastal areas. Changes in the benthic community structure can be largely correlated with the extent of organic enrichment at sewage sludge dumping grounds. Table $\mathbf{3}$ shows that Turkish coast of Black Sea's data changes.

\section{Metal Toxicity Effects to the Black Sea Ecosystem}

Metals are natural constituents of the biosphere. They occur at a wide range of concentrations and a broad array of chemical attributes. Organism absorb heavy metals, essential or not, from the surrounding environment with the potential to accumulate them within their bodies. Certain heavy metals, such as copper and zinc, are essential biological micronutrients. All heavy metals are potential toxins at some concentration, the non - essential metals e.g. mercury, lead and cadmium are particularly toxic at relatively low concentrations. Heavy metals exert toxic effects at some concentration that have metal remnant in their wastes and by non-point source surface runoff [12]. It is well known that 
Table 2. Participation of Pollutants Load from Turkish Coast of the Black Sea [9]

\begin{tabular}{|c|c|c|}
\hline No & Stations & Total Load (t.yr $\left.{ }^{-1}\right) \%$ \\
\hline 1 & Samsun & 20.6 \\
\hline 2 & Trabzon & 39.1 \\
\hline 3 & Giresun & 56.0 \\
\hline 4 & Zonguldak & 68.4 \\
\hline 7 & Rize & 86.1 \\
\hline 8 & Bul-Co fish plant (Giresun) & 89.3 \\
\hline 9 & Sinop & 91.3 \\
\hline 10 & Bartın & 92.9 \\
\hline 14 & Kastamonu & 96.8 \\
\hline 15 & Bolu & 97.3 \\
\hline 16 & Dogan Biscuit and Chocolate (Sakarya) & 97.8 \\
\hline 17 & SEKA Paper Plant (Zonguldak) & 98.1 \\
\hline 18 & Caroglu Slaughterhouse (Sakarya) & 98.3 \\
\hline 19 & Koy-Tur Chicken Plant (Sakarya) & 98.5 \\
\hline 20 & Mankap Vegetable Liquid Oil Plant (Zonguldak) & 98.7 \\
\hline 21 & Karsu fish bait Plant (Trabzon) & 98.8 \\
\hline 28 & Elif Flour Plant (Trabzon) & 99.4 \\
\hline 29 & Cargill Flour Plant (Sakarya) & 99.5 \\
\hline 30 & Ketas Food Make with Milk Plant (Trabzon) & 99.5 \\
\hline 31 & Ak A. Textile Plant (Zonguldak) & 99.6 \\
\hline 32 & Ahenk Turkish Delight and Sugaring Plant (Samsun) & 99.7 \\
\hline 33 & Kebir Food Make with Milk Plant (Trabzon) & 99.7 \\
\hline 35 & Ansan Beverages Plant (Trabzon) & 99.3 \\
\hline 34 & Camadan Flour Plant (Samsun) & 99.3 \\
\hline 36 & Ünsan Flour Plant (Ordu) & 99.9 \\
\hline 37 & Entas Chicken Plant (Sakarya) & 99.9 \\
\hline 38 & Terme Metal Industry Plant (Samsun) & 99.9 \\
\hline 39 & Yıldız Resine Plant (Samsun) & 99.9 \\
\hline 40 & Özkasapoglu Feed Plant (Zonguldak) & 100.0 \\
\hline 41 & Bizon Lumber Plant (Sakarya) & 100.0 \\
\hline
\end{tabular}

non-essential metals are very toxic to animals, so that the metal concentrations are regulated for human health and drinking water [13].
The pollution levels of the aquatic environment by heavy metals can be estimated by analyzing water, sediments and marine organisms. The levels of heavy metals in marine 


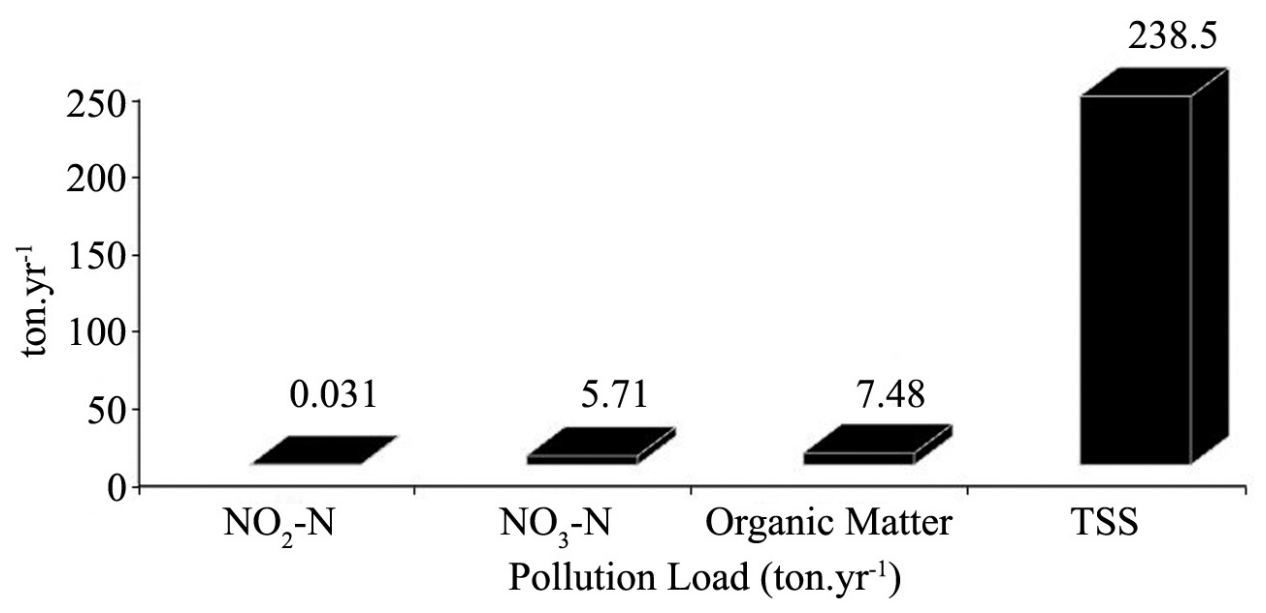

Fig. (3). Organic matter, suspended solid matter, nitrite nitrogen and nitrate nitrogen loads from Sinop city domestic discharge point [7].

organisms are often higher than in other constituents of marine environment because their ability to concentrate heavy metals from their habitat and it is important to know the changes in metal levels in marine ecosystem [13].

Metal concentrations are controlled by some solubility methods of their compounds with the ions ordinarily present in the sea. Some of the heavy metals naturally occurring in the marine environment are essential for normal growth. Thus, these organisms play a key role in the analytical schemes.

Most trace element pollution problems involve metals and rivers appear to be the most important transport mechanisms of heavy metals in the sea.

Table 4 shows that annual discharges of heavy metals in rivers and streams resulted from evaluation of inventories in the Black Sea region.

Table 3. Comparison of Data Related to Years in Black Sea Turkish Coasts (TSS: Total Suspended Solid, DO: Dissolved Oxygen)

\begin{tabular}{|l|c|c|c|c|}
\hline \multicolumn{1}{|c|}{ Comparison of July Data } & {$[\mathbf{1 0 ]}$} & {$[\mathbf{1 1}]$} & {$[\mathbf{5}]$} & {$[\mathbf{7 ]}$} \\
\hline \hline Salinity $(\% \mathbf{)}$ & 17.5 & 13.8 & & 15 \\
\hline $\mathrm{pH}$ & 7.9 & 7.15 & & 7.84 \\
\hline TSS $\left(\mathrm{mgr} . \mathrm{l}^{-1}\right)$ & 22 & & 148.99 & 656 \\
\hline DO $\left(\mathrm{mgr} . \mathrm{l}^{-1}\right)$ & 6 & 5.17 & & 2.8 \\
\hline Temperature $\left({ }^{0} \mathrm{C}\right)$ & 26.2 & 22.81 & & 25 \\
\hline Organic matter $\left(\mathrm{mgr} . \mathrm{l}^{-1}\right)$ & 9 & 7.22 & & 9.12 \\
\hline Nitrate nitrogen $\left(\mathrm{mgr} . \mathrm{l}^{-1}\right)$ & 1.1 & & 1.82 & 1.43 \\
\hline Nitrite nitrogen $\left(\mathrm{mgr} . \mathrm{l}^{-1}\right)$ & 0.019 & & 0.032 & 0.04 \\
\hline
\end{tabular}

The seasonal results of heavy metal concentrations sampling points through the Sinop, Samsun and Ordu cities in the Middle Black Sea Region of Turkey during May 2000October 2001 (Table 5).

Kizilirmak and Yesilirmak, the two most important rivers of Black Sea Region, and a lot of big and little industries (food, cement, fertilizer, pesticides, resin, plastic, textile, cigarette manufacturing) exist in the Middle Black Sea Region of Turkey. Most of these factories have no treatment plant and they have potential to create local pollution problem [14]. The two important iron and steel factories of Turkey exist in the western part of the Black Sea region. On the other hand, the eastern part of the Black Sea Region has no important industrial factories, but only hazelnut facilities, floor manufacturing and fish-oil factories. Besides small industrial activities, pulp and paper factory present in this region is one of the important industries. Heavy metals in marine environment causes by especially discharge of industrial pollutants.

The Black Sea has historically been one of the most biological productive regions in the world. According to investigations, these biological speciality losses year by year with the effects of pollution in Black Sea.

Most trace element pollution problems involve metals. Terms of trace elements identified a large group of metallic elements which are present in living organisms in limited amounts. Cadmium, mercury, chrome and lead are metals without any established biological function and include the more important contaminants in aquatic environment [15].

Anthropogenic activities such as agriculture, mining, industrial processing of ores and the use of metal components has resulted in increased inputs of heavy metals into the sea. Coastal urban centres are also sources large amounts of matter introduced into marine environment.

Marine organisms can be used as monitors to give information on concentrations of heavy metal. In this review these metal concentrations were reviewed in the Turkish coastal of the Black Sea. Table 6 shows the concentration of heavy metals in marine biota collected from Turkish Black Sea coast.

Sinop Peninsula is located on the Southern coasts of the Black Sea. Balkas et al. [3] pointed out that the oceanography of the Black Sea has been relatively well documented. The same, however, cannot be said for documentation of the levels of marine pollution and the regions that are affected by various human activities, especially in coastal areas [3]. According to the available data the heavy metal pollution increased in marine organisms of the Turkish Black Sea coast during the years. The Black 
Table 4. Annual Load of Heavy Metals in Rivers and Streams Along the Black Sea Coast of Turkey [14]

\begin{tabular}{|c|c|c|c|c|}
\hline Rivers and Streams & Discharge $\left(\mathrm{km}^{3} \cdot \mathrm{yr}^{-1}\right)$ & Total Chrome (t.yr $\left.{ }^{-1}\right)$ & Cadmium (t.yr $\left.{ }^{-1}\right)$ & Lead $\left(t . y r^{-1}\right)$ \\
\hline Sakarya River & 6.02 & 128.595 & 781.144 & - \\
\hline Melen Stream & 1.57 & 95.96 & 28.726 & - \\
\hline Alapli Stream & 0.27 & - & 11.520 & 13.927 \\
\hline Kozlu Stream & 0.02 & - & 0.053 & - \\
\hline Zonguldak Stream & 0.13 & 0.791 & 0.885 & - \\
\hline Filyos Stream & 3.22 & - & 262.824 & - \\
\hline Bartin Stream & 0.36 & 16.230 & 0.773 & 18.196 \\
\hline Yesilirmak River & 10.26 & 2549.228 & 1505.295 & - \\
\hline Milic Stream & 0.43 & - & 3.067 & 0.459 \\
\hline Civil Stream & 0.6 & 3.140 & 0.117 & - \\
\hline Melet River & 0.83 & 128.298 & 124.780 & 5.045 \\
\hline Aksu Stream & 0.97 & 3.608 & 8.326 & - \\
\hline Fol Stream & 0.20 & - & - & - \\
\hline Sögütlüdere Stream & 0.12 & - & 2.633 & - \\
\hline Stream of Degirmen & 0.87 & - & - & - \\
\hline
\end{tabular}

Table 5. The Seasonal Results of Heavy Metal Concentrations of Shore and Off-Shore Sampling Points through the Sinop, Samsun and Ordu Cities in the Middle Black Sea Region of Turkey During May 2000-October 2001 [14]

\begin{tabular}{|c|c|c|c|c|c|c|c|c|c|c|c|c|c|c|c|c|c|c|c|c|c|c|c|c|}
\hline \multirow{2}{*}{$\begin{array}{l}\text { Sampling } \\
\text { Point }\end{array}$} & \multicolumn{24}{|c|}{ Sampling Date and Pollutant Level $\left(\mu \mathrm{g} . \mathrm{M}^{+2} . \mathrm{l}^{-1}\right)$} \\
\hline & Cd & $\mathbf{P b}$ & $\mathbf{Z n}$ & $\mathbf{N i}$ & $\mathbf{C u}$ & Mn & Cd & $\mathbf{P b}$ & $\mathbf{Z n}$ & $\mathbf{N i}$ & $\mathbf{C u}$ & Mn & Cd & $\mathbf{P b}$ & $\mathbf{Z n}$ & $\mathbf{N i}$ & $\mathbf{C u}$ & Mn & Cd & $\mathbf{P b}$ & $\mathbf{Z n}$ & $\mathbf{N i}$ & $\mathbf{C u}$ & Mn \\
\hline Sinop Peninsula-A & 13 & - & 42 & 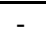 & - & 152 & 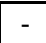 & - & 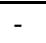 & 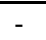 & 200 & 160 & 34 & 30 & - & - & - & - & $\mathrm{x}$ & $\bar{x}$ & $x$ & $\mathrm{x}$ & $\mathrm{x}$ & $x$ \\
\hline $\mathrm{B}$ & $\mathrm{x}$ & $\mathrm{x}$ & $\mathrm{x}$ & $\mathrm{x}$ & $\mathrm{x}$ & $\mathrm{x}$ & 4 & - & - & - & 180 & - & 50 & - & 538 & - & - & - & $\mathrm{x}$ & $\mathrm{x}$ & $\mathrm{x}$ & $\mathrm{x}$ & $\mathrm{x}$ & $\mathrm{x}$ \\
\hline Kizilirmak -A & 13 & - & 44 & - & - & 127 & - & - & - & - & 110 & - & - & 25 & - & 12 & - & - & - & 307 & - & 81 & 23 & 99 \\
\hline Kurtun Stream-A & - & - & 35 & - & - & 135 & 6 & 261 & - & - & 200 & - & 17 & - & 338 & - & - & - & - & 148 & 11 & 12 & 20 & 224 \\
\hline B & $\mathrm{x}$ & $\mathrm{x}$ & $\mathrm{x}$ & $\mathrm{x}$ & $\mathrm{x}$ & $\mathrm{x}$ & - & 250 & - & - & 250 & 140 & - & 130 & - & - & - & - & - & 67 & - & 31 & 5 & 166 \\
\hline Samsun Harbour-A & - & - & 26 & - & - & 127 & 6 & - & - & - & 200 & - & 39 & 20 & - & - & 6 & - & - & 34 & 135 & - & 26 & 163 \\
\hline $\mathrm{B}$ & $\mathrm{x}$ & $\mathrm{x}$ & $\mathrm{x}$ & $\mathrm{x}$ & $\mathrm{x}$ & $\mathrm{x}$ & - & - & - & - & 210 & - & 35 & - & 549 & 133 & 36 & 18 & - & - & 21 & 56 & - & 163 \\
\hline Mert River-KBI, TÜGSAS-A & 30 & - & 13 & - & - & 157 & - & 218 & - & - & 240 & 150 & 20 & 20 & 207 & 78 & - & 33 & - & 171 & 3 & 31 & 20 & 252 \\
\hline B & $\mathrm{x}$ & $\mathrm{x}$ & $\mathrm{x}$ & $\mathrm{x}$ & $\mathrm{x}$ & $\mathrm{x}$ & - & - & - & - & - & 40 & 41 & 10 & - & - & 12 & 56 & - & 91 & - & 10 & - & 250 \\
\hline
\end{tabular}

Sea receives large quantities of unregulated and uncontrolled fresh water with drawl for irrigation purposes, hydro and thermal power generation and the use of coastal areas for permanent human settlements; shipping; and untreated domestic, industrial and agricultural wastes drain into the sea via the rivers or directly: In spite of this, research on the heavy metal pollution in marine biota of the Black Sea is very limited. Moreover, corresponding data on the pollution state of the Black Sea off Turkey are rare.

The data presented in Table 6 were compared with the guidelines $[45,46]$ for heavy metals in fish and shellfish. 
Table 6. Heavy Metal Concentrations in Marine Biota from Sinop Coast of the Black Sea (Expressed in $\mu \mathrm{g}$ Metal $\mathrm{g}^{-1} \mathrm{Wet}_{\mathrm{wt}}$ (BS: Black Sea, EBS: Eastern Black Sea)

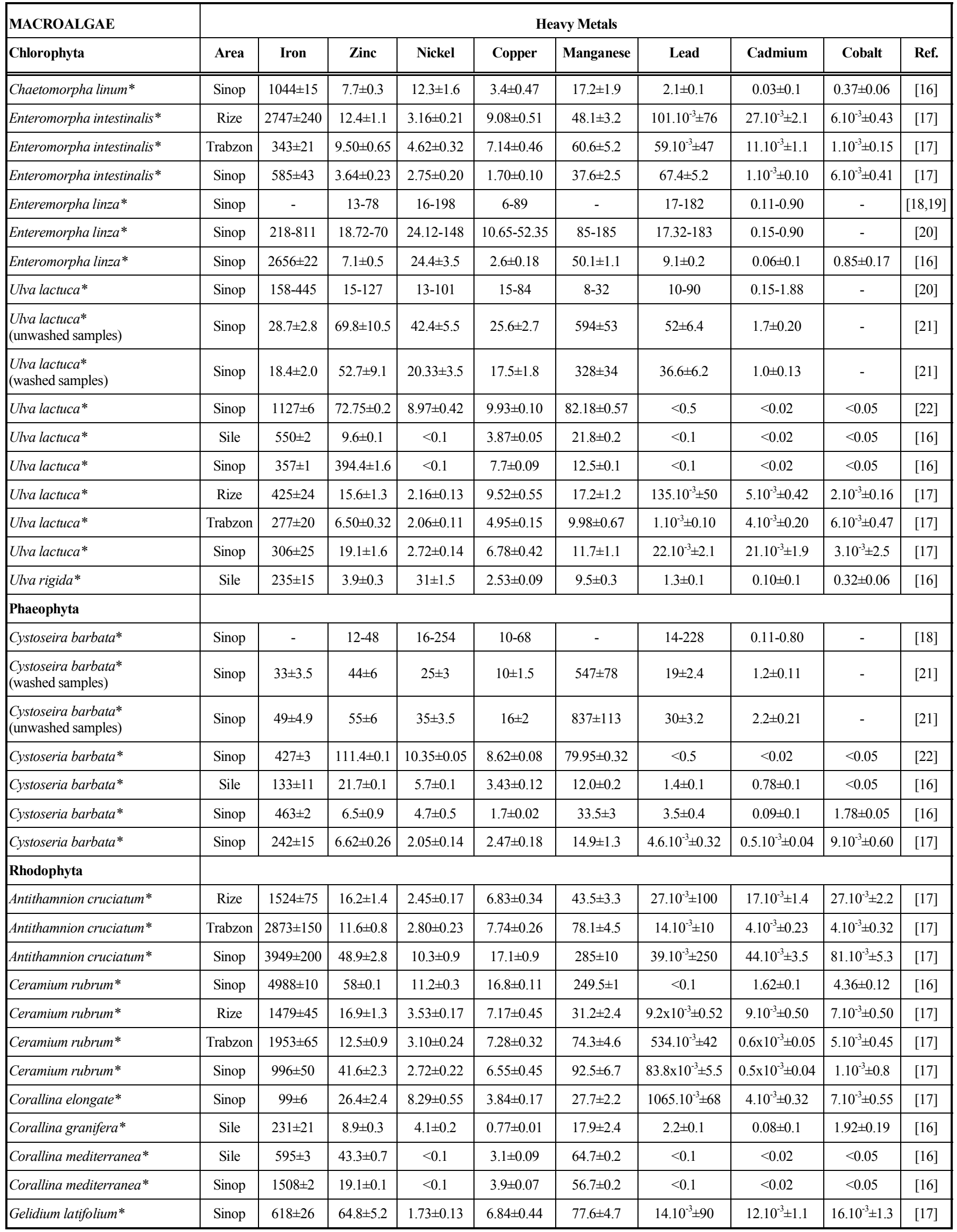


(Table 6) contd....

\begin{tabular}{|c|c|c|c|c|c|c|c|c|c|c|}
\hline \begin{tabular}{|l} 
MACROALGAE \\
Rhodophyta
\end{tabular} & \multicolumn{10}{|c|}{ Heavy Metals } \\
\hline Porphyra umbilicalis* & Rize & $784 \pm 24$ & $22.4 \pm 1.1$ & $4.04 \pm 0.15$ & $3.93 \pm 0.10$ & $19.1 \pm 1.2$ & $648.10^{-3} \pm 30$ & $23.6 \times 10^{-3} \pm 1.5$ & $42.10^{-3} \pm 3.4$ & {$[17]$} \\
\hline Porphyra umbilicalis* & Trabzon & $330 \pm 16$ & $22.8 \pm 1.2$ & $0.27 \pm 0.01$ & $4.92 \pm 0.23$ & $22.3 \pm 2.1$ & $6.1 \times 10^{-3} \pm 0.25$ & $11.4 \times 10^{-3} \pm 0.6$ & 6. $10^{-3} \pm 0.43$ & {$[17]$} \\
\hline Phyllophora nervosa* & Sinop & $1359 \pm 26$ & $54.4 \pm 0.3$ & $70.6 \pm 1.8$ & $20.1 \pm 0.12$ & $364.6 \pm 1.8$ & $<0.1$ & $<0.02$ & $9.08 \pm 0.45$ & {$[16]$} \\
\hline Phyllophora nervosa* & Sinop & $1559 \pm 65$ & $48.6 \pm 1.8$ & $36.2 \pm 2.2$ & $14.1 \pm 0.7$ & $261 \pm 17$ & $22.10^{-3} \pm 78$ & $4.10^{-3} \pm 0.33$ & $49.10^{-3} \pm 3.5$ & {$[17]$} \\
\hline Pterocladia capillacea* & Sile & $288 \pm 1$ & $86.2 \pm 0.5$ & $<0.1$ & $5.3 \pm 0.20$ & $52.1 \pm 0.2$ & $<0.1$ & $1.36 \pm 0.1$ & $<0.05$ & {$[16]$} \\
\hline Pterocladia capillacea* & Sinop & $407 \pm 5$ & $176.8 \pm 1.1$ & $<0.1$ & $<0.03$ & $10.8 \pm 0.7$ & $<0.1$ & $<0.02$ & $<0.05$ & {$[16]$} \\
\hline \multicolumn{11}{|l|}{ Angiospermae } \\
\hline $\begin{array}{l}\text { Cymodocea nodosa* } \\
\text { (washed samples) }\end{array}$ & Sinop & $626 \pm 106$ & $40.4 \pm 3.4$ & $23.7 \pm 3.66$ & $12.3 \pm 1.45$ & $157 \pm 20$ & $10.5 \pm 2.48$ & $0.08 \pm 0.02$ & - & {$[23]$} \\
\hline $\begin{array}{l}\text { Cymodocea nodosa* } \\
\text { (unwashed samples) }\end{array}$ & Sinop & $1230 \pm 148$ & $56 \pm 3.9$ & $40.3 \pm 5.2$ & $18.8 \pm 2.10$ & $219 \pm 21$ & $21 \pm 5.1$ & $0.16 \pm 0.03$ & - & {$[23]$} \\
\hline \multicolumn{11}{|l|}{ CRUSTACEA } \\
\hline Carcinus aestuarii & Sinop & $1.32-4.72$ & $3.66-7.19$ & $0.15-1.55$ & $0.17-4.40$ & $0.03-0.43$ & $0.25-0.96$ & $0.03-0.07$ & - & {$[13]$} \\
\hline Eriphia verrucosa & Sinop & $2.54 \pm 0.78$ & $10.1 \pm 1.55$ & $1.42 \pm 0.43$ & $2.61 \pm 0.38$ & $0.17 \pm 0.022$ & $0.44 \pm 0.08$ & $0.18 \pm 0.041$ & - & {$[24]$} \\
\hline Idotea baltica & Sinop & $2.02-8.21$ & $11.12-17.93$ & $4.28-10.19$ & $5.12-8.71$ & $14.81-29.12$ & $0.29-0.91$ & $0.28-0.82$ & - & {$[20]$} \\
\hline Idotea baltica & Sinop & $4.1 \pm 1.12$ & $14 \pm 1.38$ & $7.7 \pm 0.71$ & $6.7 \pm 0.68$ & $21.9 \pm 3.26$ & $0.61 \pm 0.09$ & $0.60 \pm 0.09$ & - & {$[23]$} \\
\hline \multicolumn{11}{|l|}{ MOLLUSC } \\
\hline Mytilus galloprovincialis & Sakarya & - & - & - & $0.17-0.56$ & - & $0.0-0.02$ & - & - & {$[25]$} \\
\hline Mytilus galloprovincialis & Zonguldak & - & - & - & $0.33-3.63$ & - & $0.1-0.84$ & - & - & {$[25]$} \\
\hline Mytilus galloprovincialis & Sinop & - & $1.58-7.28$ & - & $0.10-1.89$ & - & $0.11-1.18$ & $0.03-0.27$ & - & {$[26]$} \\
\hline Mytilus galloprovincialis* & Amasra & $355 \pm 1$ & $512.5 \pm 2.6$ & $4.17 \pm 0.25$ & $7.26 \pm 0.02$ & $10.11 \pm 0.05$ & $2.60 \pm 1.1$ & $6.44 \pm 0.01$ & $2.68 \pm 0.11$ & {$[22]$} \\
\hline Mytilus galloprovincialis* & Sinop & $598 \pm 7$ & $256.4 \pm 1.3$ & $4.02 \pm 0.19$ & $8.01 \pm 0.02$ & $22.8 \pm 0.11$ & $0.31 \pm 0.19$ & $1.79 \pm 0.01$ & $1.79 \pm 0.01$ & {$[22]$} \\
\hline Mytilus galloprovincialis* & Rize & $511 \pm 3$ & $78.12 \pm 0.15$ & $24.07 \pm 0.26$ & $11.52 \pm 0.02$ & $5.66 \pm 0.07$ & $<0.05$ & $<0.02$ & $5.36 \pm 0.33$ & {$[22]$} \\
\hline Mytilus galloprovincialis* & Samsun & - & - & - & - & - & $1.085 \pm 0.065$ & 0.41 & - & [27] \\
\hline Mytilus galloprovincialis* & Sinop & - & - & - & - & - & $0.26 \pm 0.03$ & $0.47 \pm 0.01$ & - & {$[27]$} \\
\hline Mytilus galloprovincialis* & Sinop & - & $24.862-519.701$ & - & $4.301-10.96$ & - & - & $0.305-4.878$ & - & {$[28]$} \\
\hline Mytilus galloprovincialis* & Samsun & - & 317.25 & 43.8 & 23.35 & 46.9 & 0.95 & $<0.02$ & - & {$[12]$} \\
\hline Mytilus galloprovincialis* & Samsun & - & 328.05 & $<0.05$ & 13.1 & 66.35 & $<0.05$ & $<0.02$ & - & {$[12]$} \\
\hline Mytilus galloprovincialis* & Samsun & - & 396.5 & 0.6 & 12.85 & 73.05 & 108.6 & $<0.02$ & - & [12] \\
\hline Mytilus galloprovincialis* & Samsun & - & 312.15 & 2.55 & 11.75 & 49.15 & 14.7 & $<0.02$ & - & {$[12]$} \\
\hline Mytilus galloprovincialis* & Camburnu & $3340 \pm 165$ & $630 \pm 32$ & $6.0 \pm 0.3$ & $190 \pm 6$ & $59 \pm 3$ & $21.0 \pm 1.0$ & $4.0 \pm 0.2$ & - & [29] \\
\hline Mytilus galloprovincialis* & Rize & $2390 \pm 72$ & $600 \pm 30$ & $1.0 \pm 0.1$ & $260 \pm 8$ & $54 \pm 3$ & $5.0 \pm 0.3$ & $3.0 \pm 0.2$ & - & {$[29]$} \\
\hline Mytilus galloprovincialis* & Rize & $1400 \pm 42$ & $340 \pm 10$ & $3.0 \pm 0.2$ & $90 \pm 3$ & $41 \pm 2$ & $9.0 \pm 0.5$ & $3.0 \pm 0.2$ & - & [29] \\
\hline
\end{tabular}


(Table 6) contd.....

\begin{tabular}{|c|c|c|c|c|c|c|c|c|c|c|}
\hline MOLLUSC & \multicolumn{10}{|c|}{ Heavy Metals } \\
\hline Patella caerula & Sinop & - & $0.128-0.770$ & $0.111-1.944$ & $0.142-0.998$ & - & $0.265-2.625$ & $0.042-0.391$ & - & {$[19]$} \\
\hline Patella caerula & Sinop & $3.81-9.62$ & $0.25-0.95$ & $0.76-1.90$ & $0.23-0.92$ & $7.73-15.38$ & $0.49-2.72$ & $0.19-0.48$ & - & {$[20]$} \\
\hline Patella caerula & Sinop & $3.19-8.84$ & $1.43-4.72$ & $0.61-2.31$ & $0.47-1.64$ & $0.12-0.58$ & $0.02-0.057$ & $0.02-0.049$ & - & {$[31]$} \\
\hline Rapana venosa & Sinop & - & $0.215-0.84$ & $0.259-0.604$ & $0.214-1.603$ & - & $0.260-0.979$ & $0.156-0.550$ & - & {$[32]$} \\
\hline Rapana venosa & Fatsa & $199 \pm 37$ & $49 \pm 6$ & $2.17 \pm 0.6$ & $57 \pm 8$ & $1.9 \pm 0.8$ & $3.2 \pm 1.2$ & $1.0 \pm 0.5$ & - & {$[33]$} \\
\hline Rapana venosa (muscle)* & Persembe & $98 \pm 1$ & $44.6 \pm 0.1$ & $<0.01$ & $35.02 \pm 0.14$ & $3.48 \pm 0.03$ & $<0.5$ & $0.37 \pm 0.03$ & $0.3 \pm 0.05$ & {$[22]$} \\
\hline \multicolumn{11}{|l|}{ FISHES } \\
\hline Alosa bulgarica & Sinop & $1.61-9.14$ & $1.65-4.48$ & $0.84-2.73$ & $0.26-0.52$ & $0.18-0.44$ & $0.18-0.74$ & $0.19-0.47$ & - & {$[34]$} \\
\hline Alosa bulgarica (liver) & Sinop & $9.14 \pm 1.307$ & $4.48 \pm 4.16$ & $2.73 \pm 0.34$ & $0.52 \pm 0.072$ & $0.44 \pm 0.052$ & $0.74 \pm 0.125$ & $0.47 \pm 0.080$ & - & {$[24]$} \\
\hline Alosa bulgarica (muscle) & Sinop & $1.61 \pm 0.307$ & $1.65 \pm 0.17$ & $0.84 \pm 0.202$ & $0.26 \pm 0.056$ & $0.18 \pm 0.032$ & $0.18 \pm 0.028$ & $0.19 \pm 0.056$ & - & {$[24]$} \\
\hline Alosa caspia* & Samsun & $16.08 \pm 1.15$ & $20.41 \pm 1.75$ & - & $2.93 \pm 0.18$ & $1.57 \pm 0.24$ & $0.52 \pm 0.16$ & $0.35 \pm 0.05$ & - & {$[35]$} \\
\hline Belone belone (muscle) & Sinop & $25 \pm 4.1$ & $7.76 \pm 1.37$ & $1.22 \pm 0.14$ & $0.54 \pm 0.05$ & $0.95 \pm 0.15$ & $0.51 \pm 0.08$ & $0.05 \pm 0.007$ & - & {$[13]$} \\
\hline Clupea sprattus* & Samsun & $25.48 \pm 3.18$ & $9.50 \pm 0.60$ & - & $1.79 \pm 0.062$ & $2.82 \pm 0.24$ & $0.74 \pm 0.11$ & $0.30 \pm 0.15$ & - & {$[35]$} \\
\hline Engraulis encrasicolus & Inebolu & - & - & - & $0.68-1.33$ & - & $0.06-0.06$ & - & - & {$[25]$} \\
\hline Engraulis encrasicolus* & Samsun & $10.45 \pm 1.63$ & $17.38 \pm 2.01$ & - & $1.94 \pm 0.10$ & $1.96 \pm 0.12$ & $0.38 \pm 0.02$ & $0.20 \pm 0.03$ & - & {$[35]$} \\
\hline Engraulis encrasicolus* & BS & $95.6 \pm 8.1$ & $40.2 \pm 3.2$ & $2.63 \pm 0.15$ & $0.95 \pm 0.08$ & $5.61 \pm 0.40$ & $0.33 \pm 0.01$ & $0.65 \pm 0.04$ & - & {$[38]$} \\
\hline $\begin{array}{l}\text { Engraulis encrasicolus } \\
\text { (muscle) }\end{array}$ & Trabzon & $44.4 \pm 9.23$ & $10.8 \pm 1.29$ & $1.51 \pm 0.26$ & $0.88 \pm 0.10$ & $0.76 \pm 0.13$ & $0.12 \pm 0.03$ & $0.03 \pm 0.01$ & $0.07 \pm 0.03$ & {$[40]$} \\
\hline $\begin{array}{l}\text { Engraulis encrasicolus } \\
\text { (liver) }\end{array}$ & Trabzon & $188 \pm 76.9$ & $14.1 \pm 2.31$ & $2.87 \pm 0.78$ & $1.08 \pm 0.20$ & $1.11 \pm 0.20$ & $0.47 \pm 0.13$ & $0.07 \pm 0.02$ & $0.19 \pm 0.05$ & {$[40]$} \\
\hline $\begin{array}{l}\text { Engraulis encrasicolus } \\
\text { (muscle) }\end{array}$ & Sinop & $35.7 \pm 9.81$ & $10.6 \pm 0.88$ & $0.63 \pm 0.19$ & $1.12 \pm 0.16$ & $0.70 \pm 0.12$ & $0.27 \pm 0.05$ & $0.02 \pm 0.00$ & $0.06 \pm 0.01$ & {$[40]$} \\
\hline $\begin{array}{l}\text { Engraulis encrasicolus } \\
\text { (liver) }\end{array}$ & Sinop & $78 \pm 11.5$ & $12.5 \pm 0.96$ & $5.10 \pm 0.59$ & $1.27 \pm 0.20$ & $1.53 \pm 0.36$ & $0.74 \pm 0.19$ & $0.06 \pm 0.01$ & $0.11 \pm 0.02$ & {$[40]$} \\
\hline $\begin{array}{l}\text { Engraulis encrasicolus } \\
\text { (muscle) }\end{array}$ & Bartın & $35.9 \pm 12.1$ & $45.6 \pm 22.1$ & $0.51 \pm 0.12$ & $8.58 \pm 2.15$ & $2.82 \pm 1.12$ & $0.87 \pm 0.40$ & $0.06 \pm 0.02$ & $0.08 \pm 0.01$ & {$[40]$} \\
\hline $\begin{array}{l}\text { Engraulis encrasicolus } \\
\text { (liver) }\end{array}$ & Bartın & $124 \pm 19.9$ & $145 \pm 38$ & $1.19 \pm 0.10$ & $30.7 \pm 7.54$ & $9.67 \pm 2.65$ & $3.38 \pm 0.55$ & $0.24 \pm 0.09$ & $0.53 \pm 0.19$ & {$[40]$} \\
\hline Engraulis encrasicolus* & BS & $18.0 \pm 2.697$ & $25.416 \pm 3.664$ & $0.34 \pm 0.106$ & - & $1.390 \pm 0.326$ & $0.329 \pm 0.302$ & $0.124 \pm 0.018$ & - & {$[39]$} \\
\hline $\begin{array}{l}\text { Merlangius merlangus } \\
\text { euxinus }\end{array}$ & Sinop & - & - & - & $0.12-2.00$ & - & $0.033-1.76$ & - & - & {$[41]$} \\
\hline $\begin{array}{l}\text { Merlangius merlangus } \\
\text { euxinus (liver) }\end{array}$ & Sinop & $18.68 \pm 2.98$ & $9.18 \pm 1.98$ & $5.12 \pm 0.61$ & $1.87 \pm 0.11$ & $2.29 \pm 0.38$ & $1.81 \pm 0.07$ & $0.110 \pm 0.009$ & - & {$[37]$} \\
\hline $\begin{array}{l}\text { Merlangius merlangus } \\
\text { euxinus (muscle) }\end{array}$ & Sinop & $9.04 \pm 1.52$ & $4.36 \pm 0.71$ & $2.61 \pm 0.51$ & $0.88 \pm 0.08$ & $1.20 \pm 0.14$ & $0.74 \pm 0.06$ & $0.025 \pm 0.004$ & - & {$[37]$} \\
\hline
\end{tabular}


(Table 6) contd...

\begin{tabular}{|c|c|c|c|c|c|c|c|c|c|c|}
\hline FISHES & \multicolumn{10}{|c|}{ Heavy Metals } \\
\hline $\begin{array}{l}\text { Merlangius merlangus } \\
\text { euxinus* }\end{array}$ & EBS & 14.137 & 15.322 & - & 2.71 & - & 1.078 & 0.601 & - & {$[42]$} \\
\hline $\begin{array}{l}\text { Merlangius merlangus } \\
\text { euxinus* }\end{array}$ & Samsun & - & - & - & - & - & $<0.05$ & $<0.02$ & - & {$[27]$} \\
\hline $\begin{array}{l}\text { Merlangius merlangus } \\
\text { euxinus* (muscle) }\end{array}$ & Sinop & 57.2 & 38.47 & 0.312 & 18.54 & 0.675 & 2.184 & 0.355 & - & {$[31]$} \\
\hline $\begin{array}{l}\text { Merlangius merlangus } \\
\text { euxinus* }\end{array}$ & Sinop & - & $8.862-163.277$ & - & $0.913-8.952$ & - & - & - & - & {$[28]$} \\
\hline Merlangus merlangus & Kastamonu & - & - & - & $0.62-3.25$ & - & $0.02-0.11$ & - & - & {$[25]$} \\
\hline Merlangus merlangus* & Rize & $46 \pm 1$ & $30.2 \pm 0.1$ & $<0.01$ & $4.54 \pm 0.11$ & $2.22 \pm 0.04$ & $<0.05$ & $<0.02$ & $<0.05$ & {$[22]$} \\
\hline Merlangus merlangus* & BS & 2.5 & 3.3 & - & 1.3 & - & 0.088 & 0.0131 & - & [43] \\
\hline Merlangus merlangus* & BS & $104 \pm 9.8$ & $48.6 \pm 3.9$ & $1.92 \pm 0.10$ & $1.25 \pm 0.10$ & $1.96 \pm 0.10$ & $0.93 \pm 0.07$ & $0.55 \pm 0.04$ & - & {$[38]$} \\
\hline Merlangus merlangus* & BS & $4.48 \pm 0.441$ & $6.029 \pm 0.545$ & $1.36 \pm 0.50$ & - & $0.07 \pm 0.024$ & $0.502 \pm 0.104$ & $0.192 \pm 0.02$ & - & {$[39]$} \\
\hline Mugil cephalus* & BS & $82.7 \pm 5.6$ & $40.2 \pm 3.3$ & $5.68 \pm 0.40$ & $1.26 \pm 0.10$ & $4.21 \pm 0.24$ & $0.61 \pm 0.04$ & $0.45 \pm 0.03$ & - & {$[38]$} \\
\hline Mugil spp. (muscle)* & Sinop & 231.5 & 104.4 & 14.52 & 13.22 & 109.3 & 1.367 & 0.183 & - & {$[31]$} \\
\hline Mugil spp.* (liver) & Sinop & 308.3 & 95.73 & 7.688 & 62.39 & 9.55 & 0.183 & 0.365 & - & {$[31]$} \\
\hline Mullus barbatus* & Trabzon & $39.0 \pm 1.0$ & $11.5 \pm 3.5$ & - & $9.10 \pm 5.9$ & $0.40 \pm 0.13$ & $6.86 \pm 0.26$ & $<0.1$ & $0.06 \pm 0.03$ & {$[44]$} \\
\hline Mullus barbatus* & BS & 4.5 & 4.3 & - & 0.01 & - & 0.077 & 0.017 & - & [43] \\
\hline Mullus barbatus* & Sinop & -- & $1.424-63.290$ & - & $0.380-2.714$ & - & - & - & - & [28] \\
\hline Mullus barbatus* & BS & $163 \pm 12$ & $106 \pm 9.1$ & $4.34 \pm 0.35$ & $0.98 \pm 0.07$ & $6.54 \pm 0.50$ & $0.84 \pm 0.07$ & $0.45 \pm 0.04$ & - & {$[38]$} \\
\hline Mullus barbatus* & BS & $21.2 \pm 1.476$ & $7.573 \pm 0.389$ & $0.658 \pm 0.33$ & - & $0.005 \pm 0.018$ & $0.727 \pm 0.141$ & $0.208 \pm 0.017$ & - & {$[39]$} \\
\hline Mullus surmelutus* & Sinop & $21.3 \pm 4.3$ & $28.0 \pm 9.0$ & - & $4.20 \pm 1.8$ & $0.42 \pm 0.13$ & $<0.5$ & $0.42 \pm 0.09$ & $0.32 \pm 0.08$ & {$[44]$} \\
\hline Platichthys flesus & & - & - & - & - & - & $<0.05$ & $0.88 \pm 0.01$ & - & {$[27]$} \\
\hline Pomatomus saltator (muscle) & Sinop & $21 \pm 3.7$ & $9.40 \pm 1.48$ & $1.20 \pm 0.09$ & $0.58 \pm 0.08$ & $0.96 \pm 0.16$ & $0.55 \pm 0.08$ & $0.05 \pm 0.004$ & - & [13] \\
\hline Pomatomus saltator* (meat) & Sinop & 421.3 & 82.2 & 20.22 & 35.6 & 69.02 & 2.253 & 0.343 & - & {$[31]$} \\
\hline Pomatomus saltator* & BS & $68.6 \pm 5.3$ & $35.4 \pm 3.2$ & $3.89 \pm 0.30$ & $1.83 \pm 0.10$ & $1.28 \pm 0.10$ & $0.38 \pm 0.02$ & $0.60 \pm 0.05$ & - & {$[38]$} \\
\hline Psetta maxima* & Trabzon & $31.0 \pm 1.7$ & $38.6 \pm 4.1$ & - & $4.2 \pm 0.6$ & $1.25 \pm 0.39$ & $2.38 \pm 0.09$ & $0.30 \pm 0.07$ & $0.59 \pm 0.16$ & {$[44]$} \\
\hline Psetta maxima* & Igneada & $46.7 \pm 26.3$ & $19.7 \pm 2.5$ & - & $6.8 \pm 4.2$ & $0.48 \pm 0.15$ & $1.47 \pm 0.05$ & $0.57 \pm 0.13$ & $1.31 \pm 0.36$ & {$[44]$} \\
\hline Psetta maxima* (liver) & Sinop & 373.6 & 125.8 & 25.98 & 14.22 & 126.1 & 1.037 & 0.268 & - & {$[31]$} \\
\hline Psetta maxima* (meat) & Sinop & 113.3 & 170.5 & 23.32 & 26.14 & 125.4 & 2.72 & 0.272 & - & {$[31]$} \\
\hline Raja clavata* & Sinop & - & $6.601-35.873$ & - & $0.496-9.356$ & - & - & - & - & [28] \\
\hline Sarda sarda* & Samsun & $9.52 \pm 0.81$ & $11.20 \pm 1.44$ & - & $1.28 \pm 0.14$ & $1.06 \pm 0.27$ & $0.22 \pm 0.04$ & $0.09 \pm 0.02$ & - & [35] \\
\hline Sarda sarda* & BS & $73.5 \pm 6.3$ & $48.7 \pm 3.7$ & - & $0.84 \pm 0.05$ & $2.68 \pm 0.22$ & $0.76 \pm 0.05$ & $0.90 \pm 0.07$ & - & {$[38]$} \\
\hline
\end{tabular}




\begin{tabular}{|c|c|c|c|c|c|c|c|c|c|c|}
\hline FISHES & Area & Iron & Zinc & Nickel & Copper & Manganese & Lead & Cadmium & Cobalt & Ref. \\
\hline Scomber scombrus* (meat) & Sinop & 120.3 & 69.53 & 1.684 & 3.62 & 9.44 & 2.948 & 0.172 & - & {$[31]$} \\
\hline Scopthalmus maeoticus* & Sinop & - & - & - & - & - & $<0.05$ & $<0.02$ & - & {$[27]$} \\
\hline Solea vulgaris* (viscera) & Sinop & 127 & 67.97 & 2.553 & 29.72 & 4.621 & 2.006 & 0.504 & - & {$[31]$} \\
\hline Spicara smaris* & Sinop & - & $6.234-57.743$ & - & $0.610-4.161$ & - & - & - & - & {$[28]$} \\
\hline Spicara smaris (muscle) & Trabzon & $32.2 \pm 8$ & $12.2 \pm 2.63$ & $0.25 \pm 0.07$ & $0.83 \pm 0.10$ & $0.39 \pm 0.05$ & $0.15 \pm 0.04$ & $0.02 \pm 0.00$ & $0.04 \pm 0.01$ & {$[40]$} \\
\hline Spicara smaris (liver) & Trabzon & $75.7 \pm 14.8$ & $18.5 \pm 2.38$ & $5.71 \pm 1.04$ & $1.86 \pm 0.22$ & $0.72 \pm 0.09$ & $1.01 \pm 0.19$ & $0.23 \pm 0.07$ & $0.08 \pm 0.03$ & {$[40]$} \\
\hline Trachurus trachurus (liver) & Sinop & $14.71 \pm 1.86$ & $4.16 \pm 1.09$ & $3.92 \pm 0.65$ & $1.38 \pm 0.09$ & - & $1.36 \pm 0.38$ & $0.050 \pm 0.007$ & - & {$[37]$} \\
\hline Trachurus trachurus (muscle) & Sinop & $4.28 \pm 0.95$ & $3.28 \pm 0.66$ & $1.57 \pm 0.26$ & $0.79 \pm 0.06$ & $0.47 \pm 0.06$ & $0.74 \pm 0.21$ & $0.028 \pm 0.002$ & - & {$[37]$} \\
\hline Trachurus trachurus* & Samsun & $32.40 \pm 2.70$ & $12.05 \pm 2.30$ & - & $1.52 \pm 0.35$ & $3.76 \pm 0.45$ & $0.85 \pm 0.16$ & $0.47 \pm 0.10$ & - & {$[35]$} \\
\hline Trachurus trachurus* & BS & $74.3 \pm 6.1$ & $37.4 \pm 2.9$ & $3.93 \pm 0.25$ & $0.95 \pm 0.04$ & $7.40 \pm 0.60$ & $0.68 \pm 0.05$ & $0.50 \pm 0.03$ & - & {$[38]$} \\
\hline \multicolumn{11}{|l|}{ MAMMALS } \\
\hline Dolphin* (muscle) & Trabzon & $10.1 \pm 0.6$ & $6.5 \pm 0.3$ & $<0.35$ & $0.72 \pm 0.10$ & $0.06 \pm 0.02$ & $1.50 \pm 0.05$ & $<0.10$ & $<0.10$ & {$[36]$} \\
\hline Dolphin* (liver) & Trabzon & $561 \pm 71$ & $84.1 \pm 32.8$ & $<0.35$ & $8.35 \pm 0.49$ & $3.87 \pm 1.22$ & $2.13 \pm 0.08$ & $0.99 \pm 0.22$ & $<0.10$ & {$[36]$} \\
\hline
\end{tabular}

- : not measured, *: expressed in $\mu \mathrm{g}$ metal $\mathrm{g}^{-1}$ dry wt.

From the public health point of view, the levels of the metals found in these studies are generally lower than the permitted levels (Table 7).

According to Marine General Quality Criteria given in Turkish Environmental Regulation (Table 7), it was seen that $\mathrm{Cd}^{2+}$ and $\mathrm{Cu}^{2+}$ levels generally were exceeded the criterion.
In case of $\mathrm{Pb}^{2+}$ and $\mathrm{Zn}^{2+}$ levels they were sometimes exceeded the criterion, while $\mathrm{Ni}^{2+}$ concentrations were at the desired levels (see Table 5).

In the available data of land-based pollution comprising river, stream, shore and harbor were shown to be exposed the heavy metal pollution [14].

Table 7. Some Part of General Quality Criteria of Marine and Inland Water Sources According to the Classes (I: High Quality Water, II: Slightly/Moderately Polluted Water, III: Polluted Water, IV: Heavily Polluted Water) [47]

\begin{tabular}{|c|c|c|c|c|c|}
\hline Water Quality Parameters & $\mathbf{I}$ & II & III & IV & Marine (mg. $\left.\left.\right|^{-1}\right)$ \\
\hline DO & - & - & - & - & $<90 \%$ of saturation \\
\hline SS & - & - & - & - & 30 \\
\hline T. Phosphorus ( $\mu \mathrm{g}$ PO4 $4^{-3}-\mathrm{P} .1^{-1)}$ & 20 & 160 & 650 & $>650$ & - \\
\hline Cadmium $\left(\mu \mathrm{g} \mathrm{Cd}^{+2} \cdot 1^{-1}\right)$ & 3 & 5 & 10 & $>10$ & 0.01 \\
\hline Lead $\left(\mu \mathrm{g} \mathrm{Pb}^{+2} \cdot 1^{-1}\right)$ & 10 & 20 & 50 & $>50$ & 0.1 \\
\hline Copper $\left(\mu \mathrm{g} \mathrm{Cu}{ }^{+2} \cdot 1^{-1}\right)$ & 20 & 50 & 200 & $>200$ & 0.01 \\
\hline T. Chromium $\left(\mu \mathrm{g} \mathrm{Cr}^{+2} \cdot 1^{-1}\right)$ & 20 & 50 & 200 & $>200$ & 0.1 \\
\hline Manganese $\left(\mu \mathrm{g} \mathrm{Mn}{ }^{+2} \cdot 1^{-1}\right)$ & 100 & 500 & 3000 & $>3000$ & - \\
\hline
\end{tabular}




\section{CONCLUSION}

Sustainable development of the Black Sea requires continued international co-operation. Solutions to the Black Sea environmental problems demand that uniform strict rules be adopted by each country. It means that the regulations should also cover those countries which influence the Black Sea environment through the rivers, mainly Danube, Dnieper and Dniester and another land based pollution sources.

Different types of pollutants in domestic and/or industrial discharges have different effects on human health and ecosystems at the point of discharge and in the surrounding environment. This surrounding environment may be very large and may extend beyond international borders. The risks increase proportionally with the quantity of the wastewater and concentration of the pollutant. Turkey is developing countries where industrial and urban developments mostly occur in coastal areas through increased input of wastes impose a further stress on the Turkish coasts of Black Sea.

The application of the agreements requires that each country which has a coast to the Black Sea, concerned creates an environmental policy. Harmonization of legislation and standards, preparation of effluent discharge inventories and mapping of major pollution sources and establishment of water monitoring programmers. These components are stated in the activities of the Black Sea Environmental Programme but the legislative frame for their realization still does not exist in all countries in the region.

\section{REFERENCES}

[1] Clark RB. Marine pollution. Oxford: Clarendon Press 1986.

[2] Clark RB. Marine pollution. $3^{\text {rd }}$ ed. Oxford: Clarendon Press 1992.

[3] Balkas T, Dechev G, Mihnea R, Serbanescu O, Ünlüata U. State of the marine environment in the Black Sea Region. UNEP Regional Seas Reports and Studies 1990; No. 124: p. 41.

[4] Bakan G, Özkoç HB, Büyükgüngör H, Ergun ON, Onar N. Evaluation of the Black Sea and- based sources inventory results of the coastal region of Turkey. Proceedings of the International Workshop on MED \& Black Sea ICZM: Sarıgerne, Turkey 1996; pp. 39-52.

[5] Bakan G, Büyükgüngör H. The Black Sea. Marine Poll Bull 2000; 41: 24-43.

[6] Gökkurt O, Bat L, Sahin F. The investigation of some physicochemical parameters in the Middle Black Sea (Sinop. Turkey). Proceedings of $7^{\text {th }}$ National Environmental Engineering Congress. İzmir, Turkey 2007; pp: 869-73 (in Turkish).

[7] Gökkurt O. The effects of potential sewage points to the water quality and the organisms on the coast of Sinop. Master Thesis. Samsun: Ondokuz Mayis University, Institute of Science 2007; p. 202 (in Turkish).

[8] Anonym. Sinop directory of environmental and forestry. Sinop Environmental Condition Report 2004; 2005: p. 95 (in Turkish).

[9] Sarıkaya HZ, Sevimli MF, Citil E. Region-wide assessment of the land-based sources of pollution of the Black Sea. Water Sci Technol 1999; 39(9.8):193-200.

[10] Anonym. Karadeniz Bölgesi'nde su kirliliğine sebep olan faktörlerin belirlenmesi ve su ürünlerine etkilerinin araştırılması. T. C. Tarım ve Köyişleri Bakanlığı, Su Ürünleri Araștırma Enstitüsü Müdürlügü, Trabzon. Sinop: Turkey 1996; p. 175 (in Turkish).

[11] Bat L, Gündoğdu A, Sezgin M, Çulha M, Gönlügür G, Akbulut M. Acute toxicity of zinc, copper and lead to three species of marine organisms from the Sinop Peninsula, Black Sea. Turk J Biol 1998; 23:537-44.

[12] Bakan G, Özkoç HB. An ecological risk assessment of the impact of heavy metals in surface sediments on biota from the mid-Black Sea coast of Turkey. Int J Environ Stud 2007; 64 (Pt 1): 45-57.

[13] Bat L, Öztürk M, Öztürk M. Heavy metal concentrations in some fish and crab from the Black Sea of Turkey. II. Spil Fen Bilimleri Dergisi (Biyoloji), 23-25 Ekim 1997 Manisa, Celal Bayar
Üniversitesi Fen -Edebiyat Fak. Dergisi 1998; 1: 148-55 (in Turkish).

[14] Altas L, Büyükgüngör H. Heavy metal pollution in the Black Sea shore and offshore of Turkey. Environ Geol 2006; DOI 10.2007/s00254-006-0480-1.

[15] Viarengo A. Biochemical effects of trace metals. Mar Pollut Bull 1985; 16: 153-7.

[16] Topcuoğlu S, Güven KC, Balkış N, Kırbaşoğlu Ç. Heavy metals monitoring of marine algae from the Turkish Coast of the Black Sea, 1998-2000. Chemosphere 2003; 52 (10): 1683-8.

[17] Tuzen M, Verep B, Ogretmen AO, Soylak M. Trace element content in marine algae species from the Black Sea, Turkey. Environ Monit Assess 2009; 151:363-8.

[18] Öztürk M. A Study on the two invertebrata and two algae species for the their heavy metal build up on their respective levels those tend to live in Sinop Province's inner and outer harbors or coves, O.M.Ü. Fen Bilimleri Enstitüsü: Doktora Tezi 1991; pp:85 (in Turkish).

[19] Öztürk M. Heavy metal levels in Patella coerulae L. and Enteremorpha linza (L.) J. Ag. collected from Sinop bay and harbour. Turk J Biol 1994; 18: 195-211 (in Turkish).

[20] Öztürk M, Bat L, Öztürk M. Heavy metal levels in bioindicator species collected from Sinop bay and harbour. Proceedings of the $7^{\text {th }}$ National Biology Congress. Edirne-Turkey: Trakya Üniversitesi Fen-Ed Fak 1994; vol. 2: pp. 20-5 (in Turkish).

[21] Öztürk M, Öztürk M, Bat L. Comparison of the heavy metal accumulation levels in washed and unwashed samples of two algae species distributed on Sinop coasts of the Black Sea. J Fish Aquat Sci 1996;13 (3 Pt 4): 409-23 (in Turkish).

[22] Topcuoğlu S, Kırbaşoğlu Ç, Güngör N. Heavy metals in organisms and sediment from Turkish Coast of the Black Sea, 1997-1998. Environ Int 2002; 27: 521-6.

[23] Bat L, Öztürk M. Heavy metal levels in some organisms from Sinop Peninsula of the Black Sea. Turk J Eng Environ Sci 1997; 21: 29-33.

[24] Öztürk M, Bat L. Levels of trace elements in some edible organisms in the Sinop coast of the Black Sea. J Fish Aquat Sci 1994; 16(1): 177-96 (in Turkish).

[25] Ünsal M, Bekiroğlu Y, Akdoğan Ş, et al. Determination of heavy metals in some economically important marine organisms in southwestern Black Sea. TUBITAK Project No: DEBAG-80/G 1993; pp:78 (in Turkish).

[26] Bat L, Gündoğdu A, Öztürk M, Öztürk M. Copper, zinc, lead and cadmium concentrations in the Mediterranean mussel Mytilus galloprovincialis Lamarck, 1819 from the Sinop coasts of the Black Sea. Turk J Zool 1999; 23: 321-6.

[27] Das YK, Aksoy A, Baskaya R, Duyar HA, Güvenc D, Boz V. Heavy metal levels of some marine organisms collected in Samsun and Sinop Coasts of Black Sea, in Turkey. J Anim Vet Adv 2009; 8 (3): 496-99.

[28] Turk CS, Bat L, Culha M, Efendioglu A, Andac M, Bati B. Heavy metals levels in some fishes and molluscs from Sinop Peninsula of the Southern Black Sea, Turkey. Rapp Comm Int Mer Medit 2007; 38: 323 .

[29] Çevik U, Damla N, Kobya AI, et al. Assessment of metal element concentrations in mussel (M. galloprovincialis) in Eastern Black Sea, Turkey. J Hazard Mater 2008;160: 396-401.

[30] Bat L, Öztürk M, Öztürk M. Patella caerula as a biomonitor of coastal metal pollution. II. Spil Fen Bilimleri Serisi (Biyoloji), 2325 Ekim 1997, Celal Bayar Üniversitesi Fen-Ed. Fak. Dergisi 1998; 1: 142-7 (in Turkish).

[31] Bat L, Gündoğdu A, Yardım Ö, Zoral T, Çulha S. Heavy metal amounts in zooplankton and some commercial teleost fish from inner harbour of Sinop, Black Sea. SÜMDER (Su Ürünleri Müh. Dergisi) 2006; 25: 22-7 (in Turkish).

[32] Öztürk M, Öztürk M. Heavy metal levels in sea snail (Rapana venosa Valenciennes, 1846) collected from Sinop bay and harbour. Turk J Zool 1994; 18: 193-8 (in Turkish).

[33] Topçuoğlu S, Erentürk N, Esen N, et al. Toxic element levels in oyster and sea snail. E. Ü. Fen Fakültesi Dergisi 1994; 16 (1): 239241 (in Turkish).

[34] Bat L. A Study on trace element levels in some organisms living in the upper - infralittoral zone of Sinop peninsula. Ondokuz Mayis Ünv. Fen Bil. Enst., Su Ürünleri ABD. Master thesis, Sinop 1992; p.108 (in Turkish). 
[35] Tüzen M. Determination of heavy metals in fish samples of the middle Black Sea (Turkey) by graphite furnace atomic absorption spectrometry. Food Chem 2003; 80: 119-23.

[36] Topçuoğlu S, Kut D, Erentürk N, Esen N, Saygı N. Some element levels in anchovy, bluefish, Atlantic mackerel and dolphin. Turk J Eng Environ Sci 1995;19: 307-10 (in Turkish).

[37] Bat L, Öztürk M. Öztürk M. Heavy metal amounts in some commercial teleost fish from the Black Sea. O.M.Ü. Faculty of Science-Arts. J Sci 1996; 7(1): 117-35.

[38] Uluozlu OD, Tuzen M, Mendil D, Soylak M. Trace metal content in nine species of fish from the Black and Aegean Seas, Turkey. Food Chem 2007; 104: 835-40.

[39] Turan C, Dural M, Oksuz A, Öztürk B. Levels of heavy metals in some commercial fish species captured from the black sea and mediterranean coast of Turkey. Bull Environ Contam Toxicol 2009; 82: 601-4.

[40] Türkmen A, Tepe Y, Türkmen M. Metal levels in tissues of the European anchovy, Engraulis encrasicolus L, 1758, and picarel, Spicara smaris L, 1758, from Black, Marmara and Aegean Seas. Bull Environ Contam Toxicol 2008; 80: 521-5.

[41] Ünsal M, Doğan M, Ataç Ü, et al. Determination of heavy metals in the marine organisms of economical importance in the central and eastern Black Sea. Tarım ve Köyişleri Bakanlığı Trabzon Su
Ürünleri Araştırma Enstitüsü. Project No: DEBAG-18/G 1992; pp: 52 (in Turkish).

[42] Boran M, Karaçam H, Çelikkale S, Köse S, Kutlu S. Levels of heavy metals in blue whiting caught from the eastern Black Sea area of Turkey. Toxicol Environ Chem 2000; 75: 67-73.

[43] Dalman Ö, Demirak A, Balc1 A. Determination of heavy metals $(\mathrm{Cd}, \mathrm{Pb})$ and trace element $(\mathrm{Cu}, \mathrm{Zn})$ in sediment and fish of the Southeastern Aegean Sea (Turkey) by Atomic Absorption Spectrometry. Food Chem 2006; 95: 157-62.

[44] Topçuoğlu S, Erentürk N, Saygı N, et al. Trace metal levels of fish from the Marmara and Black Sea. Toxicol Environ Chem 1990; 29: 95-9.

[45] MAFF. Monitoring and surveillance of non-radioactive contaminants in the aquatic environment and activities regulating the disposal of wastes at sea, 1993, Directorate of Fisheries research, Lowestoft, Aquatic Environment Monitoring Report 1995; No.44.

[46] The Food Safety (Live Bivalve Molluscs and Other Shellfish). (Import Conditions and Miscellaneous Amendments) Regulations. Statutory Instrument 1994 No. 2782. 1994; Available from: http://www.opsi.gov.uk/SI/si1994/Uksi_19942782_en_1.htm

[47] Turkish Environmental Regulations Water Pollution Control Regulation [word document on the Internet] 2004-2005; Available from: http: www.cevreorman.gov.tr/yasa/yonetmelik.asp

(C) Bat et al.; Licensee Bentham Open.

This is an open access article licensed under the terms of the Creative Commons Attribution Non-Commercial License (http://creativecommons.org/licenses/by$\mathrm{nc} / 3.0 /$ ) which permits unrestricted, non-commercial use, distribution and reproduction in any medium, provided the work is properly cited. 